\title{
Granulomatosis with Polyangiitis Presenting as Cholangitis and Acute Kidney Injury
}

\author{
Jong Hwan Jung and Seon-Ho Ahn* \\ Division of Nephrology, Department of Internal Medicine, Wonkwang University School of Meidicine and Hospital, Iksan, Korea
}

A 74-year-old male visited the emergency department for a few days of dyspepsia and abdominal pain unresponsive to digestive medicine. He was febrile, and laboratory tests had following results: white blood cells, $16210 / \mu \mathrm{L}$; blood urea nitrogen, $62.8 \mathrm{mg} / \mathrm{dL}$; serum creatinine, $4.30 \mathrm{mg} / \mathrm{dL}$; alkaline phosphatase, $887 \mathrm{U} / \mathrm{L}$; direct bilirubin, $1.47 \mathrm{mg} / \mathrm{dL}$; gamma glutamyl transferase, 165 IU/L; amylase, 1115 IU/L; lipase, 1161 IU/L; C-reactive protein, $176.97 \mathrm{mg} / \mathrm{L}$. Although he did not show any pulmonary symptoms, computed tomography (CT) showed only edematous changes around the common bile duct (CBD) (Fig. 1A) and reticular opacity showing a suspicious pattern of interstitial lung disease or collagen vascular disease in both lower lobes (Fig. 1B). Magnetic resonance cholangiopancreatography (MRCP) showed CBD dilatation and tiny renal cysts (Fig. 1C). His symptoms improved after antibiotics and endoscopic nasobiliary drainage. Despite appropriate therapy, serum creatinine increased to 8.50 $\mathrm{mg} / \mathrm{dL}$ after two weeks, and other laboratory results were as follows: c-anti-neutrophil cytoplasmic antibody (ANCA) and p-ANCA, 1521.95 and 14.48 AAU; antinuclear antibody (ANA), negative. We initiated hemodialysis for con- trol of uremic symptoms, and performed a renal biopsy. The pathologic results showed granuloma formation with Langerhans giant cells and fibrous crescent (Fig. 2A). Granulomatosis with polyangiitis (GPA) was diagnosed. We performed high dose-steroids and cyclophosphamide treatments concurrently with hemodialysis. Two months later, follow-up magnetic resonance imaging (MRI) was performed to identify the CBD. Interestingly, as compared to the previous MRCP, contrast-enhanced nodular lesions with increased number and size were seen in the kidneys (Fig. 2B), and a contrast-enhanced lesion was seen in the pancreatic head around CBD (Fig. 2C). The nodular lesions on the kidney turned out to be arterial aneurysms (Fig. 2D). The immunosuppressive agents and hemodialysis were continued, but pneumonia developed, and progressed to sepsis and the patient died. GPA is a systemic vasculitis characterized as necrotizing granulomatous inflammation. GPA can involve systemic organs. ${ }^{1}$ GPA mainly presents as a phenotype of rapidly progressive glomerulonephritis in glomeruli. ${ }^{2}$ However, as GPA of relatively long duration can lead to medium-sized arterial aneurysms. ${ }^{3}$ Acute cholangitis might result from the pancreatic pseudoaneurysm
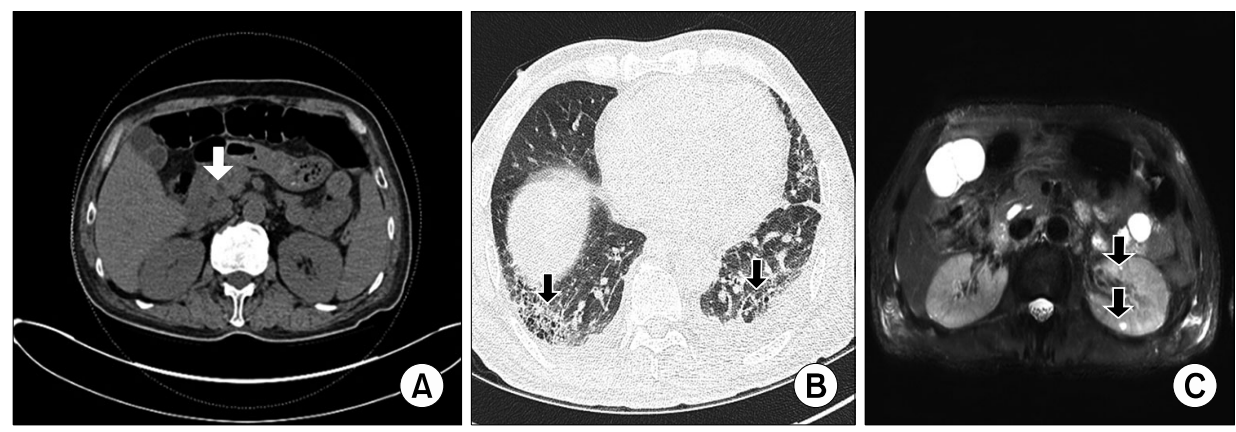

FIG. 1. Computed tomography showed only edematous change and central hypo-dense lesion around common bile duct and pancreatic head portion (white arrow) (A), and high resolution computed tomography showed reticular opacity with diffuse ground glass opacity at subpleural portion in both lower lobe and lingular segment of left upper lobe (black arrows) (B). T2-weighted image of magnetic resonance cholangiopancreatography showe distended gallbladder and dilated common bile duct and several tiny renal cysts (black arrows) (C).

Corresponding Author:

Seon-Ho Ahn

Division of Nephrology, Department of Internal Medicine, Wonkwang University School of Medicine, 895 Muwang-ro, Iksan 54538, Korea

Tel: +82-63-859-2622, Fax: +82-63-855-2025, E-mail: chjh0502@wku.ac.kr
Article History:

Received April 1, 2019

Revised April 16, 2019

Accepted April 17, 2019 

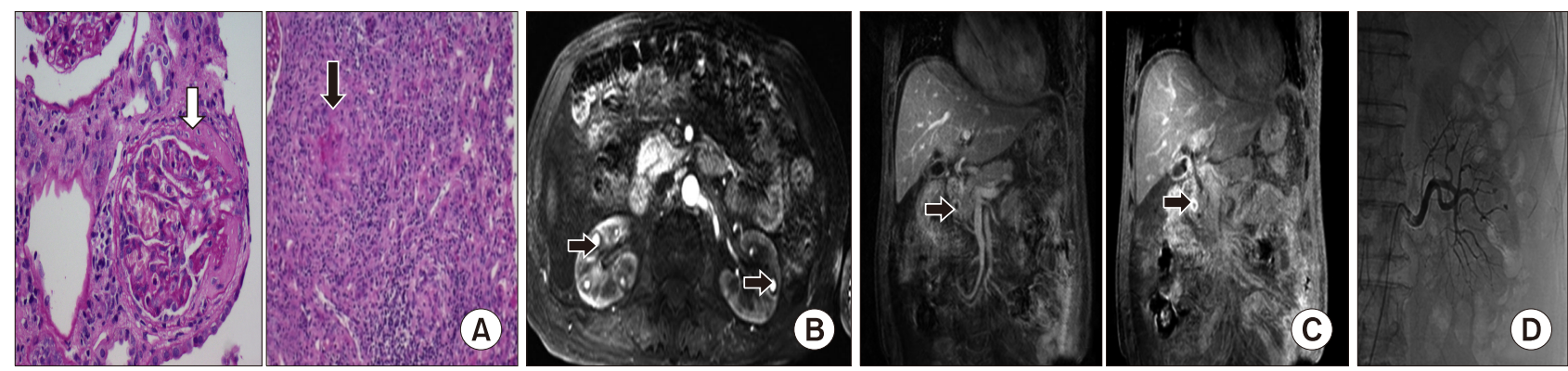

FIG. 2. The pathologic finding of the kidney demonstrates fibrous crescent and diffuse mesangial proliferation (Periodic Acid Schiff stain, $\times 400$ ) (white arrow) and markedly increased interstitial inflammation including granulomatous formation (Hematoxylin and Eosin stain, $\times 100$ ) (black arrow) (A). Follow-up T1-weighted image of magnetic resonance imaging (MRI) showed contrast-enhanced nodular lesion with various sizes on the both kidney (black arrows) (B). Nodular lesion with low signal intensity on T1-weighted MRI and high signal intensity on contrast-enhanced T1-weigthed MRI in the pancreatic head portion around common bile duct was shown (black arrows) (C). Renal artery angiography shows multiple microaneurysms on the interlobular or arcuate arteries without rupture (D).

around CBD. The pancreatic pseudoaneurysm in followup, contrast-enhanced MRI may be caused by chronic GPA in this patient. Clinically, this involvement of GPA may develop various manifestations. Therefore, if the patient is clinically suspected of having a systemic vasculitis, such as GPA, nephrologists or clinicians should consider whether clinical symptoms or signs of GPA can be manifested in various ways.

\section{ACKNOWLEDGEMENTS}

This work was supported by Wonkwang University fund in 2019. '

\section{CONFLICT OF INTEREST STATEMENT}

None declared.

\section{REFERENCES}

1. Ahn SH, Jung JH. Renal involvement in rheumatic diseases. J Rheum Dis 2017;24:174-84.

2. Zheng Z, Ding J, Li X, Wu Z. Gastric presentation (vasculitis) mimics a gastric cancer as initial symptom in granulomatosis with polyangiitis: a case report and review of the literature. Rheumatol Int 2015;35:1925-9.

3. Arlet JB, Le Thi Huong D, Marinho A, Cluzel P, Wechsler B, Piette JC. Arterial aneurysms in Wegener's granulomatosis: case report and literature review. Semin Arthritis Rheum 2008;37:265-8. 